\title{
Decriminalize Drug-Checking Technologies in Pennsylvania to Prevent Overdose Deaths
}

\section{Kyla Mace $1,5,7$, Wisberty J. Gordián-Vélez ${ }^{2,5,7}$, Aerin Wheeler ${ }^{3,5-7}$, Victor Acero ${ }^{2,5-7}$, Emily Cribas $^{4-7}$}

1Perelman School of Medicine at the University of Pennsylvania, Pharmacology Graduate Group, Philadelphia, PA

2University of Pennsylvania, Department of Bioengineering, Philadelphia, PA

3University of Pennsylvania, Department of History and Sociology of Science, Philadelphia, PA

${ }^{4}$ Perelman School of Medicine at the University of Pennsylvania, Cell and Molecular Biology Program, Philadelphia, PA

5University of Pennsylvania, Penn Students for Sensible Drug Policy, Philadelphia, PA

6University of Pennsylvania, Penn Society for Psychedelic Science, Philadelphia, PA

7University of Pennsylvania, Penn Science Policy and Diplomacy Group, Philadelphia, PA

http://doi.org/10.38126/ISPG170210

Corresponding author: kmace@pennmedicine.upenn.edu

Keywords: drug overdose; fentanyl test strips; drug-checking technologies (DCTs); people who use drugs (PWUD); Pennsylvania Controlled Substance, Drug, Device, and Cosmetic Act

\begin{abstract}
Executive Summary: Drug-checking technologies (DCTs), such as fentanyl test strips, prevent fatal overdoses caused by the adulteration of drugs. DCTs are currently classified as illegal drug paraphernalia in Pennsylvania (PA), but the recent and significant rise in fentanyl-related overdoses has prompted the PA House and Senate to propose exempting fentanyl test strips from the definition of drug paraphernalia. While fentanyl is currently the leading drug adulterant, other substances may be used to alter drugs. Therefore, we argue that broader reform is required to protect Pennsylvanians against all potential drug adulterants. We recommend that the PA House and Senate Judiciary Committees work to amend the PA Controlled Substance, Drug, Device, and Cosmetic Act to exempt all DCTs from the definition of drug paraphernalia. This proactive intervention will save countless lives by preventing adulterant-related overdoses.
\end{abstract}

\section{The overdose epidemic}

\section{i. Health costs}

The overdose crisis has dramatically impacted public health and the economy in PA. The Commonwealth had 4,491 fatal overdoses in 2018: the third-highest number of overdose deaths in the country. Every 2 hours, someone in PA dies of an overdose, and most of these deaths involve opioids (CDC 2018). Moreover, for every death, it is estimated that around 30 non-fatal overdoses occur (Frazier et al. 2017). In 2017, Philadelphia alone saw 8,065 emergency room visits related to non-fatal overdoses and sustained increases in the number of hospitalizations for overdose-related medical complications such as seizures, pneumonia, brain injury, and organ failure (Philadelphia Department of Public Health 2020; Zibbel et al. 2019).

\section{ii. Economic costs}

Beyond its health and welfare implications, the overdose crisis is expensive. Opioid overdose-related hospitalizations can cost as much as $\$ 20,500$ per patient requiring intensive care (Premier, Inc. 2019). In 2016, overdose-related fatalities, medical services, addiction treatment, criminal justice proceedings, and loss of productivity cost the Commonwealth of PA an estimated $\$ 53.8$ billion (DEA Philadelphia Division 2018). Loss of productivity is unsurprising given that 25 to 54 -year-olds, who overwhelmingly 
contribute to the workforce in PA, are at the greatest risk for overdose (DEA Philadelphia Division 2018). In addition, from 1999 to 2013, the overdose-related economic burden on state Medicaid programs totaled around \$72.4 billion, with costs increasing over time (Leslie et al. 2019). At the federal level, Medicaid recipients and Medicare patients with disabilities have 3- and 5-fold higher risk of opioid overdoses, respectively (Frazier et al. 2017; Kuo, Raji, and Goodwin 2019). Thus, overdoses collectively represent a significant financial and public health crisis.

\section{iii. Fentanyl adulteration}

The rise in fatal drug overdoses has been driven by the increasing presence of adulterants, such as extremely potent synthetic opioids like fentanyl, which was implicated in $70 \%$ of overdose deaths in PA in 2018 (OverdoseFreePA 2018). Fentanyl was originally added to opioids like heroin to increase potency, but it has also begun appearing in nonopioid drugs like cocaine and methamphetamine (Nolan et al. 2019; Zibbell et al. 2019; NDEWS 2018). Because the illicit drug market is unregulated, people who use drugs (PWUD) often do not know the purity of what they consume, putting them at risk of fentanyl-related overdose.

\section{iv. Other adulterants}

While fentanyl is present in most overdoses in PA today, other adulterants will likely take its place tomorrow. A new synthetic opioid called isotonitazene, with a similar potency to fentanyl, has already been implicated in roughly 200 overdose deaths since August 2019 (Power 2020). Ultimately, the introduction of novel adulterants in the drug supply is inevitable. Thus, legislative foresight is required to properly address these future public health crises.

\section{Legal and political landscape}

\section{i. Fentanyl test strips}

Fentanyl test strips are a type of DCT that screen for fentanyl adulteration. These strips are inexpensive (\$1-2 each), easy to use, and highly reliable (96-100\% accuracy) (Sherman et al. 2018). Recently, public health organizations have started using test strips to prevent overdoses, and studies have found that PWUD want to use them to adjust their behaviors and minimize their risk of overdose (Sherman et al. 2018;
Goldman et al. 2019; Vancouver Coastal Health 2017). Given the prevalence of fentanyl-related overdoses, fentanyl test strips are an essential intervention to address the overdose crisis (Sherman et al. 2018).

Despite their efficacy, fentanyl test strips and all DCTs are illegal in PA due to their classification as drug paraphernalia under the Controlled Substance, Drug, Device, and Cosmetic Act (The Controlled Substances, Drugs, Device, and Cosmetic Act 1972). Currently, there are two bills under consideration in the PA House and Senate Judiciary Committees, PA H.B. 1741 and PA S.B. 886, which propose an exemption to the definition of drug paraphernalia specifically for fentanyl test strips. If passed, these bills would have a real, immediate impact on the lives of PWUD in PA but would not address additional adulterants that will inevitably enter illicit drug supplies.

\section{ii. Drug-checking technologies}

Public health officials have identified the illegality of DCTs as a barrier to the implementation of drugchecking at a systemic level (Sherman et al. 2018). Other states have recently amended their definitions to allow for increased access to DCTs, as shown in Figure 1. In Illinois, a bill providing for syringe access programs also incorporated the use of DCTs (Bush et al. 2019). Maryland made DCTs legal by altering their definition of paraphernalia. DC decriminalized possession and distribution of DCTs (Bonds et al. 2017). PA has seen more total fatal drug overdoses

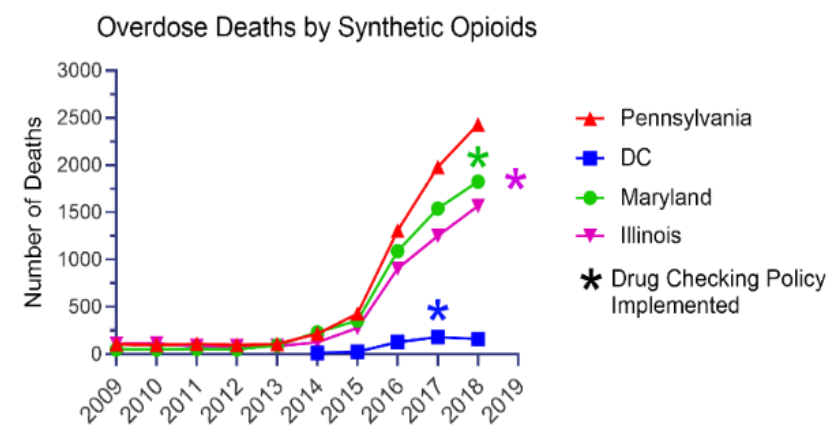

Figure 1: Overdose deaths due to synthetic opioids and year of DCT policy reform are shown by state. DCT policy reform has been implemented in states with fewer overdose deaths than PA. Data obtained from the CDC Wide-ranging Online Data for Epidemiological Research (WONDER) database, with the underlying cause of death being overdose by drug poisoning and the multiple cause of death code corresponding to synthetic narcotics other than methadone (CDC 2019). 
than Illinois, Maryland, or DC, making it even more essential that PA officials promptly take preventative measures against accidental overdoses (CDC 2018).

As evidenced in Figure 1, DCT reform has severely lagged behind the rise of fentanyl-related overdose deaths, even though there are no documented harms from the legalization of DCTs. Similarly, the time needed to introduce, pass, and enact individual DCT legislation for other adulterants will result in an unnecessary loss of Pennsylvanian lives. Instead, PA, like other states, should exempt all DCTs from the definition of drug paraphernalia.

\section{Policy options}

i. Option 1: Pass H.B. 1741/S.B. 886 to exempt fentanyl test strips under the PA Controlled Substance, Drug, Device, and Cosmetic Act.

These bills would explicitly exempt fentanyl test strips from being classified as drug paraphernalia and have bipartisan sponsorship. Fentanyl adulteration in the drug supply contributes significantly to the overdose crisis (OverdoseFreePA 2018) and an exemption for fentanyl test strips would allow PWUD to check their supply for fentanyl adulteration and take necessary precautions to ensure their safety.

\section{Advantages}

H.B. 1741 and S.B. 886 are currently under consideration in the Judiciary committees with bipartisan sponsorship. This amendment is timely and has the political momentum to pass due to the urgent need for action against overdoses. The legislation would provide immediate access to lifesaving technologies for PWUD and help attenuate fentanyl-related overdose deaths.

\section{Disadvantages}

This option does not enable PWUD to protect themselves against other known or novel adulterants. Legislative responses are likely to lag behind public health crises and will allow preventable deaths.

ii. Option 2: Remove references to "testing" and "analyzing" from the PA Controlled Substance, Drug, Device, and Cosmetic Act to legalize all DCTs.

The decriminalization of all DCTs is a proactive approach that would enable PWUD to safely screen their drugs for adulteration with fentanyl and any other substances to protect themselves from increased risks of non-fatal or fatal overdoses. Legislation providing an exemption for all DCTs has been enacted in various states including Maryland, DC, and Illinois. We propose the amendment found in the Appendix, eliminating any mention of "testing" or "analyzing" in the definition of drug paraphernalia.

\section{Advantages}

This approach would save lives and help prevent significant economic costs in future drug crises that may arise from other contaminants without a delay in legislative responses.

\section{Disadvantages}

While a fentanyl test strip-only exemption is already targeted in H.B. 1741/S.B. 886, legislation decriminalizing all DCTs has not yet been introduced. Limited political capital may make enacting our proposal more challenging. In addition, without effective regulation, DCTs might provide inaccurate readings for novel adulterants and may produce a false sense of security for PWUD.

\section{iii. Option 3: Inaction}

The PA House and Senate Judiciary Committees may indefinitely delay consideration of HB1741/SB886 or any additional measures to combat adulterantrelated drug overdoses, thus maintaining the current definition of drug paraphernalia under the PA Controlled Substance, Drug, Device, and Cosmetic Act. All DCTs would remain illegal with no exceptions for fentanyl test strips.

\section{Advantages}

It may be politically beneficial to disregard HB1741/SB886 to avoid appearing lenient on drugrelated issues. This would also alleviate concerns over encouraging drug use or providing a false sense of security to PWUD.

\section{Disadvantages}

Without introduction of alternative interventions for the overdose crisis, overdoses due to the adulterated drug supply will not be reduced and will likely increase. These consequences will be potentiated by the ongoing COVID-19 pandemic (American Medical Association 2020; Azpiri 2020). 


\section{Recommendations}

We strongly recommend Option 2: removing any reference to "testing" and "analyzing" in the definition of drug paraphernalia in the Controlled Substance, Drug, Device, and Cosmetic Act to decriminalize the possession and use of all DCTs. This will contribute to reducing fentanyl overdoses and allow for the use of DCTs immediately in the event of future overdose crises. This proactive, comprehensive reform will prevent countless overdoses that arise from current and future contaminants and adulterants in the illicit drug supply. In addition to this reform, we also urge the PA General Assembly to pass H.B. 1741 and S.B. 886 (Option 1) to provide immediate access to fentanyl test strips.

Appendix: Proposed amendment to the PA Controlled Substance, Drug, Device, and Cosmetic Act.

"Drug paraphernalia" means all equipment, products and materials of any kind which are used, intended for use or designed for use in planting, propagating, cultivating, growing, harvesting, manufacturing, compounding, converting, producing, processing, preparing, testing, analyzing, packaging, repackaging, storing, containing, concealing, injecting, ingesting, inhaling or otherwise introducing into the human body a controlled substance in violation of this act. It includes, but is not limited to: [...]

(4) Testing equipment used, intended for use or designed for use in identifying or in analyzing the strength, effectiveness or purity of controlled substances.

References

American Medical Association. 2020. "Issue Brief: Reports of Increases in Opioid-Related Overdose and Other Concerns during COVID Pandemic," 7.

An Act amending the act of April 14, 1972 (P.L.233, No.64), known as The Controlled Substance, Drug, Device and Cosmetic Act, further providing for definitions., H.B. 1741, P.A. General Assembly, Regular Session 2019-2020 (P.A. 2019)

An Act amending the act of April 14, 1972 (P.L.233, No.64), known as The Controlled Substance, Drug, Device and Cosmetic Act, further providing for definitions., S.B. 886, P.A. General Assembly, Regular Session 2019-2020 (P.A. 2019)

Azpiri, Jon. 2020. "Vancouver Sees Spike in Overdose Deaths amid COVID-19 Crisis." Global News, April 1, 2020.

https://globalnews.ca/news/6763146/vancouve r-sees-spike-in-overdose-deaths-amid-covid-19crisis/

Bonds, Anita, Mary Cheh, Jack Evans, Vincent Gray, David Grosso, Kenyan McDuffie, Phil Mendelson, et al. 2017. Opioid Abuse Treatment Act of 2017.

Bush, Melinda, Julie Morrison, Patricia Van Pelt, Robert Peters, Sue Rezin, Laura Fine, Mattie Hunter, et al. 2019. Needle and Hypodermic Access.

CDC. 2018. "National Center for Health Statistics: Drug Overdose Mortality by State." 2018. https://www.cdc.gov/nchs/pressroom/sosmap/ drug_poisoning mortality/drug_poisoning.htm

DEA Philadelphia Division. 2018. "The Opioid Threat in Pennsylvania." DEA.
Frazier, Winfred, Gerald Cochran, Wei-Hsuan Lo-Ciganic, Walid F. Gellad, Adam J. Gordon, Chung-Chou H. Chang, and Julie M. Donohue. 2017. "MedicationAssisted Treatment and Opioid Use Before and After Overdose in Pennsylvania Medicaid." JAMA 318 (8): 750.

https://doi.org/10.1001/jama.2017.7818

Goldman, Jacqueline E., Katherine M. Waye, Kobe A. Periera, Maxwell S. Krieger, Jesse L. Yedinak, and Brandon D. L. Marshall. 2019. "Perspectives on Rapid Fentanyl Test Strips as a Harm Reduction Practice among Young Adults Who Use Drugs: A Qualitative Study." Harm Reduction Journal 16 (1): 3. https://doi.org/10.1186/s12954-0180276-0

Kuo, Yong-Fang, Mukaila A. Raji, and James S. Goodwin. 2019. "Association of Disability With Mortality From Opioid Overdose Among US Medicare Adults." JAMA Network Open 2 (11): e1915638. https://doi.org/10.1001/jamanetworkopen.2019 .15638

Leslie, Douglas L., Djibril M. Ba, Edeanya Agbese, Xueyi Xing, and Guodong Liu. 2019. "The Economic Burden of the Opioid Epidemic on States: The Case of Medicaid." The American Journal of Managed Care 25 (13 Suppl): S243-49.

NDEWS. 2018. "National Drug Early Warning System." Percentage of Methamphetamine Deaths That Also Involved Fentanyl in Five SCS. 2018. https://ndews.umd.edu/feature/percentagemethamphetamine-deaths-also-involvedfentanyl-five-scs 
Nolan, Michelle L., Sindhu Shamasunder, Cody Colon Berezin, Hillary V. Kunins, and Denise Paone. 2019. "Increased Presence of Fentanyl in Cocaine-Involved Fatal Overdoses: Implications for Prevention." Journal of Urban Health 96 (1): 49-54. https://doi.org/10.1007/s11524-018$\underline{00343-\mathrm{z}}$

OverdoseFreePA. 2018. "Death Data Overview." OverdoseFreePA. 2018.

https://www.overdosefreepa.pitt.edu/know-thefacts/death-data-overview/

Philadelphia Department of Public Health. 2020. "NonFatal Overdose - Clinical Sites." Philadelphia Department of Public Health - Health Information Portal. 2020. https://hip.phila.gov/DataReports/Opioid/NFOC linicalSites

Power, Mike. 2020. "40 Americans Are Dying Every Month From Taking This New, Legal Opioid." Vice, March $12,2020$.

https://www.vice.com/en us/article/wxebjb/40 -americans-are-dying-every-month-from-takingthis-new-legalopioid?utm campaign=sharebutton

Premier, Inc. 2019. “Opioid Overdoses Costing U.S. Hospitals an Estimated \$11 Billion Annually." Premier, Inc. Newsroom. January 3, 2019. https://www.premierinc.com/newsroom/pressreleases/opioid-overdoses-costing-u-s-hospitalsan-estimated-11-billion-annually
Sherman, S.G., J.N. Park, J. Glick, M. McKensie, K. Morales, T. Christensen, and T.C. Green. 2018. "FORECAST Study Summary Report.” Johns Hopkins Bloomberg School of Public Health. https://americanhealth.jhu.edu/sites/default/fil es/inlinefiles/Fentanyl Executive Summary 032018.pdf

The Controlled Substances, Drugs, Device, and Cosmetic Act. 1972. Pa. C.S. Vol. 35.

Vancouver Coastal Health. 2017. "Drug Checking at Insite Shows Potential for Preventing Fentanyl-Related Overdoses." Vancouver Coastal Health. May 15, 2017. http://www.vch.ca/about-us/news/newsreleases/drug-checking-at-insite-showspotential-for-preventing-fentanyl-relatedoverdoses

Zibbel, Jon, Jennifer Howard, Sarah Duhart Clarke, Abigail Ferrel, and Sarita Karon. 2019. "Non-Fatal Opioid Overdose and Associated Health Outcomes: Final Summary Report." U.S. Department of Health and Human Services, September, 33.

Zibbell, Jon E., Arnie P. Aldridge, Dennis Cauchon, Jolene DeFiore-Hyrmer, and Kevin P. Conway. 2019. "Association of Law Enforcement Seizures of Heroin, Fentanyl, and Carfentanil With Opioid Overdose Deaths in Ohio, 2014-2017." JAMA Network Open 2 (11): e1914666. https://doi.org/10.1001/jamanetworkopen.2019 $\underline{.14666}$

Kyla Mace is a graduate student in the Pharmacology Graduate Group at the University of Pennsylvania with a research background in the neurobiology of substance use disorders. She holds a B.A. in Psychology from the University of North Carolina, Chapel Hill.

Wisberty J. Gordián-Vélez is a PhD candidate in the Bioengineering Graduate Group at the University of Pennsylvania studying neural tissue engineering approaches to treat neurodegenerative disease. He holds a B.S. in Chemical Engineering from the University of Puerto Rico, Mayagüez Campus.

Aerin Wheeler is a graduating senior at the University of Pennsylvania studying Public Health and Neuroscience. Her research interests include health disparities, healthcare delivery, and community medicine.

Victor Acero studies tissue engineering and traumatic brain injury as a PhD candidate in the Department of Bioengineering at the University of Pennsylvania. He holds a B.S. in Engineering Science from the Pennsylvania State University.

Emily S. Cribas is a PhD candidate in the Cell and Molecular Biology Graduate Group at the University of Pennsylvania studying host-pathogen interactions in the gut. She holds a B.S. in Biochemistry and Molecular Biology and Psychology minor from Penn State.

\section{Acknowledgements}

The authors are grateful for the contributions of Shannon Wolfman, Jake Hoffman, Stephen Mell, and Erin Reagan from the University of Pennsylvania in preparing this manuscript. 